\title{
Diagrammatic reasoning
}

\section{Abstraction, interaction, and insight}

\author{
K. Tylén ${ }^{\mathrm{a}, \mathrm{b}}$, R. Fusarolii, ${ }^{\mathrm{a}, \mathrm{J}}$ J.S. Bjørndahla, J. Rączaszek- \\ Leonardic ${ }^{c}$, S. Østergaard ${ }^{\mathrm{a}}$ and F. Stjernfelt ${ }^{\mathrm{d}}$ \\ ${ }^{a}$ Center for Semiotics, School of Communication and Culture, Aarhus \\ University, Denmark / ${ }^{\mathrm{b}}$ The Interacting Minds Centre, Aarhus University, \\ Denmark / ' Institute of Psychology, Polish Academy of Sciences, University \\ of Warszawa, Poland / ${ }^{\mathrm{d} H u m a n o m i c s}$ Center, Department of Arts and \\ Cultural Studies, University of Copenhagen
}

\begin{abstract}
Many types of everyday and specialized reasoning depend on diagrams: we use maps to find our way, we draw graphs and sketches to communicate concepts and prove geometrical theorems, and we manipulate diagrams to explore new creative solutions to problems. The active involvement and manipulation of representational artifacts for purposes of thinking and communicating is discussed in relation to C.S. Peirce's notion of diagrammatical reasoning. We propose to extend Peirce's original ideas and sketch a conceptual framework that delineates different kinds of diagram manipulation: Sometimes diagrams are manipulated in order to profile known information in an optimal fashion. At other times diagrams are explored in order to gain new insights, solve problems or discover hidden meaning potentials. The latter cases often entail manipulations that either generate additional information or extract information by means of abstraction. Ideas are substantiated by reference to ethnographic, experimental and historical examples.
\end{abstract}

Keywords: diagrams, reasoning, problem solving, distributed cognition, extended cognition, C.S. Peirce, abstraction

\section{Introduction}

The use of diagrammatical representations pervades many of our mundane and professional practices from visualization and communication of complex meanings to problem-solving, science and logic. Inspired by C.S. Peirce's seminal works, we introduce the notion of diagrammatic reasoning and develop it into a conceptual framework articulating the different ways in which diagrams catalyze and support 
innovative thinking processes and interpersonal communication. According to this framework diagrammatic reasoning i) relies on external structures, ii) making abstract relations perceivable, iii) and manipulable, iv) in a public space, therefore enabling collective and temporally distributed forms of thinking. These properties of diagrammatic reasoning will be explored and further articulated in a variety of contexts, from the progressive construction of scientific knowledge to the development of communication systems.

While prototypical diagrams are graphical representations, such as blueprints and maps, here we extend the notion to include any representation depicting a "skeleton-like sketch of relations" (Stjernfelt, 2000), highlighting relational properties of its referent. Crucially such representation affords manipulation and thus comes to facilitate new insights or hypotheses to be tested (Peirce, 1931, 1981, 1998). In this very broad sense, diagrams include geometrical representations, scientific schemas, algebraic notation, and even gestures, models, and language, when these are employed as manipulable representations. Diagrams are characterized by iconic properties, in that they enable additional insights on their objects, and most often involve symbolic conventions regulating the specificity of the representation: e.g. an altimetric map will follow different conventions from a road map, while iconically representing the same territory. An additional important property of diagrams is their type-token structure. All empirically occurring diagrams are individual physical objects. In the reasoning process, however, those objects are interpreted as tokens of types, which form the center of attention in that process. This implies that a selection between two classes of properties of the diagram token is undertaken. Certain properties of the token are bracketed as not pertaining to the diagram type, leaving other such properties to be focused upon as relevant. In a geometrical diagram, for instance, the color is typically not considered relevant, while the shape is preserved as pertaining to the abstract diagram type. Furthermore, the properties relevant for the type are subjected to some degree of idealization (e.g. lines are taken to be straight and without breadth). By means of such procedures, the diagram makes possible diagrammatical reasoning, that is, the observation and manipulation of abstract relations by the intermediate of diagrammatical representational tokens.

We can thus begin to highlight the way in which diagrams enable specific forms of reasoning. First, they provide an external representational support to cognitive processes. External supports have been argued to extend the mind through their affordances: They can work as external memory stores — sometimes more reliable than our biological working memory. Imagine, for instance, having to memorize the full map of a new neighborhood (A. Clark \& Chalmers, 1998). Second, diagrams might make abstract properties and relations accessible by converting them to perceivable configurations (Hutchins, 1995, 2005). The history of ideas is rich 
in examples of geometrical, mathematical and logical notations suddenly enabling the exploration of new abstract domains: for instance the invention of a notation for " 0 " radically altered the understanding of many mathematical problems. More recently, as large amounts of digitalized data have become available, the field of data visualization is blossoming, enabling new ways of finding patterns in data (Cairo, 2012; Yau, 2011).

However, diagrams allow for more than simple perception of relations, they allow for their manipulation. In this regard Peirce distinguished between corollarial and theorematic diagrammatic reasoning (Hintikka, 1983; Stjernfelt, 2014). In corollarial reasoning, the synthesis of available information into a coherent diagram suffices, and the conclusion may be directly read off of the diagram: like when using a map of the London tube to establish a travel route. In theorematic reasoning, however, the diagram is actively manipulated, for instance, by moving or adding parts according to certain rules. For instance, in demonstrating Pythagoras's theorem, the representation of a triangle is not enough: a proof is made by the addition of auxiliary lines, e.g. by drawing squares on the three sides of the triangle and grids enabling the comparison of their surface. Supported by the token-type relation, the manipulation of the diagram token makes it possible to explore new aspects or possibilities inherent in the type (as when extrapolating a graph, solving an equation, etc.). Such manipulations come in a large variety of types and levels of complexity, ranging all the way from small everyday cognitive tasks to the demanding solution of high-level problems. Furthermore, by virtue of their materiality, diagrams afford long-term ratcheting of corollarial and theorematic explorations: a repeated and even cumulative manipulation and evolution, opening for more articulated explorations of their properties and relations (A. Clark, 2006a, 2008).

By this very process, diagrams facilitate the collaboration of several individuals collectively sketching and manipulating diagrams either together or separately across space and time (Tylén, Fusaroli, Bundgaard, \& Østergaard, 2013). Indeed, material representations are potentially accessible to several people (simultaneously or in succession) thus affording powerful modes of collective thinking. When put out in the public, thoughts can be intersubjectively negotiated, elaborated and tested. The joint manipulation of public representational formats (materially instantiated models, figures and diagrams, even linguistic representations, etc.) enables participants to stimulate each other in ways that may make a group perform better - as a whole - than the mere sum of its members' contributions. In these cases, mediating structures permit the formation of socially distributed cognitive systems (Dale, Fusaroli, Duran, \& Richardson, 2013; Fusaroli, Gangopadhyay, \& Tylén, 2014; Fusaroli, Raczaszek-Leonardi, \& Tylén, 2014; Fusaroli \& Tylén, in press; Hutchins, 2010). 
In the following we will consider a variety of empirical examples of diagrammatic reasoning organized along two main types of diagram manipulation. In the first case, the referent (or type) is already given and known. The diagram manipulation is thus aiming at a redescription of the referent in a way that more optimally represents relevant features/profiles, for instance, for purposes of communication. In the second case, diagrams are manipulated to gain new insights about the referent-type. Here the referent is not exhaustively known or has features that are not trivially evident. The manipulation or experimentation on the diagram-token is thus aimed at revealing "hidden" aspects of the referent relevant to a task or situation (e.g. a proof or solution to a problem, etc.). We will show that this function of a diagram can be achieved through its potential to generate and extract information. Finally we will provide examples of how the public nature of diagrams enables cognitive processes distributed across individuals and time-scales.

\section{Diagrams as redescription for optimal profiling of information}

Information can always be represented in more than one way. For instance, when figuring out how to drive to a new place, one could print out a map of the area with the highlighted route, or a list of sequential instructions: drive straight for 500 meters, then turn right in Pleasant St, proceed for 2 kilometers, etc. Different representational formats profile information in different ways, highlighting particular aspects or perspectives on the referent. For instance, the same data may be represented in a scatterplot, a violin plot, or a box plot, progressively abstracting from individual variations of the single data points and details of their distribution according to what needs to be profiled and communicated (see Figure 1).

While one representational format profiles particular aspects of the referent, it often backgrounds other aspects. Some diagram manipulations thus set out to explore the optimal representational format to profile a particular complex of meaning. These types of manipulations might entail fully replacing one format with another. Importantly, however, the referent is known and in principle neutral to these redescriptions: the representational redescription does not entail consequences for the referent. In such cases, the diagram is not a means for the diagram-producer to explore and discover new information, but to redescribe given information in a new format for instance for the purpose of communication (Karmiloff-Smith, 1995). This constitutes one of the most widespread uses of diagrams. Although the referent object is kept stable, the new diagrammatical tokens de facto produced by re-description almost inevitably involve manipulation of the diagrammatical type re-arranging the relational properties of the represented entity. 

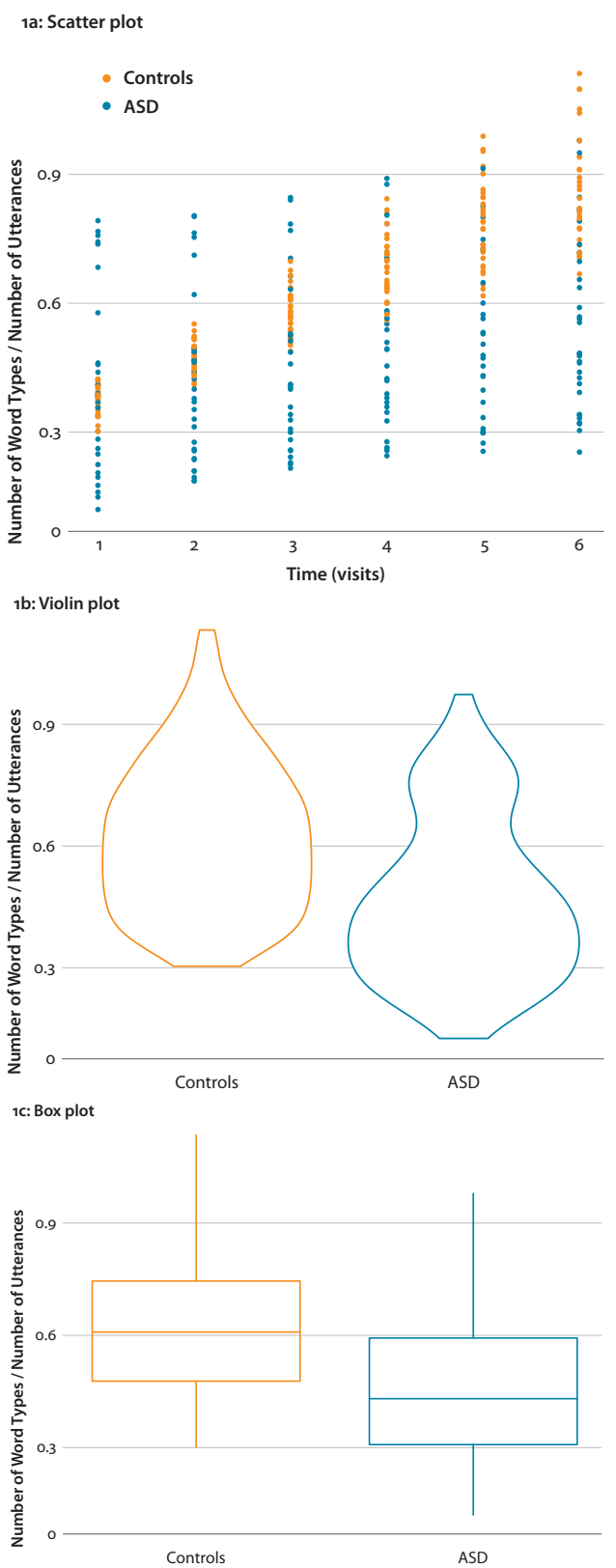

Figure 1. The three plots all represent the amount of words produced by children with Autism Spectrum Disorder and matched controls during 6 successive visits (the raw data are from Tek, Mesite, Fein, \& Naigles, 2014). Fig 1a, a scatterplot, displays the temporal progression of the two groups. Fig $1 \mathrm{~b}$, a violin plot, displays the difference in production between the two groups as well as the distribution of the values within each group. Fig $1 \mathrm{c}$, a box plot, displays the difference in production between the two groups. 
Art directors, teachers, journalists and political spin-doctors explicitly and consciously design communication by means of diagrammatical manipulation and redescription; but more spontaneous diagrams also pervade everyday discourse. Often people - without explicit reflection - recruit hand gestures, enactments, or draw figures for each other to assist verbal instruction or explanation. In a recent study, Bjørndahl and colleagues (2014; 2015) studied groups solving a series of creative tasks together involving LEGO blocks. The task was to jointly reach shared representations of abstract concepts such as "Justice", "Security" and "Collaboration". In many instances, the participants were at loss in precisely articulating their ideas using words, thus generating incomprehension and misunderstandings. In these cases, initial verbal descriptions were accompanied and followed by gestural redescriptions and exemplifications recruiting also the LEGO materials. Gestures and artifacts re-described the content of the verbal openings, making certain relational properties perceivable and more salient. Similar observations are reported in a study on how pairs of participants instructed each other in building LEGO models (H. H. Clark \& Krych, 2004). When blocking visual access so that participants could not rely upon gestures (exhibiting, poising, pointing, placing, etc.) they became significantly slower and made more errors. The visuospatial character of the task strongly afforded non-linear, iconic and deictic modes of representation, which was better met by the gestural and enactive redescriptions accompanying speech.

\subsection{The social evolution of diagrams for communication}

The exploration of representational space in order to optimize communication is an active process not limited to individual minds. In other words, these kinds of diagrammatical redescriptions are often socially distributed processes spanning multiple individuals over multiple time scales. A number of recent experimental studies have investigated how communicative representations (whether linguistic, gestural or graphic) are continuously reshaped and optimized through a history of repeated interactions among pairs or groups of people solving joint tasks (Galantucci \& Garrod, 2010). These studies experimentally create and manipulate task environments to test how different social, cognitive and environmental factors impact the evolution of representations and their functional efficacies.

An interesting example is constituted by a study utilizing a graphically mediated communication game not dissimilar to the game of Pictionary (Garrod, Fay, Lee, Oberlander, \& MacLeod, 2007). Through multiple trials, participants take turns in communicating a fixed set of categories to each other through a graphical medium. Participants are thus not allowed to use letters or other conventional signs, but have to negotiate their own graphic signs for referring to potentially 


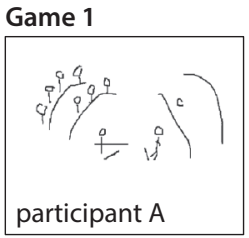

Game 4

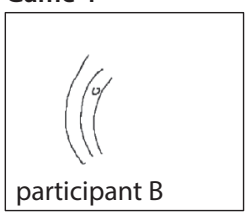

Game 2

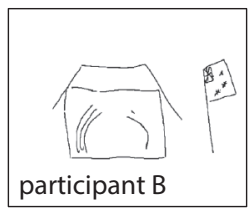

Game 5

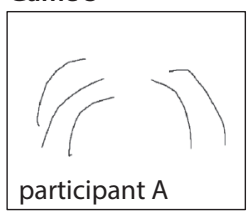

Game 3

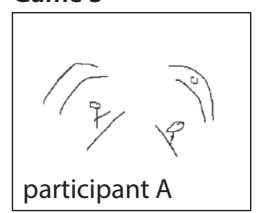

Game 6

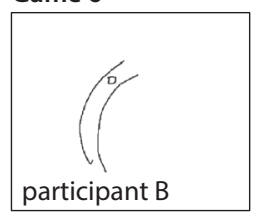

Figure 2. The interactive refinement of a graphical sign representing the concept "Parliament" negotiated between a pair of interlocutors across six games of a Pictionarylike task (Fay, Garrod, Roberts, \& Swoboda, 2010). Reprinted with permission from the authors.

confusable categories such as "cinema", "theater", and "parliament", etc. Through multiple trials participants converge from very articulated, largely iconic drawings on quite schematic and parsimonious signs differentiating the target categories in minimal and clever ways. In other words, via a process of repeated reciprocal redescriptions, participants progressively simplify the signs while refining and enhancing their discriminative relations (see Figure 2).

Interestingly, the development of more efficient, schematic and abstract signs was found to be associated with possibilities for reciprocal communication and feedback (see also Healey, Swoboda, Umata, \& King, 2007). Along similar lines, studies have found that children produce more abstract representation when working in pairs (Schwartz, 1995). Similar observations have been made for community-evolved linguistic practices in a game where participants jointly navigated a set of mazes (Garrod \& Doherty, 1994). The task was constructed so that participants depended on sharing spatial positions in differently shaped mazes consisting of interconnected boxes. Participants thus had to converge on shared reference frames for talking about positions in the two-dimensional layout. Often participants would initially use quite concrete frames of reference of the type "B: Yeah right, the extreme right it's sticking out like a sore thumb. A: That's where I am.,', relying on the particular salient shapes of the mazes. However, through repeated trials, they typically evolved increasingly abstract frames of reference that more easily carried from one maze to the next. Some participants would thus develop chess-like coordinate systems that referred to positions through row and column indices of the type "A: So you're now at D1 are you? B: Uh-huh". These 'matrix

1. Transcript examples are reprinted from Garrod and Anderson 1989 Appendix. 
schemes' also represents the highest level of abstraction from the actual mazes as the irregularly shaped mazes would often embody only a subset of the possible positions in a virtual matrix (for instance the reference position A1 could be missing in a maze). Analogously, in a joint decision task, high collective performance was associated with the ability of the participants to develop locally situated expressions of confidence into a shared, graded confidence scale, which allowed them to compare their individual levels of confidence (Fusaroli et al., 2012; Fusaroli \& Tylén, 2012).

\section{Diagram manipulation}

Whereas in the previous cases the referent of the diagram was more or less known, a second case of diagrammatical reasoning involves a more radical construction of new knowledge about the referent through diagram manipulation. A prime example of this is Copernicus' modification of the Ptolemaic model of planetary movements. The invention of the telescopic lens had made new data available, which fitted badly with Ptolemaeus' model. Copernicus made a simple rearrangement of elements from an earth-centered to a sun-centered perspective. However he still used epicycles where planets were moving on cycles, the centers of which were also cycles, etc. Later, again by actively manipulating Copernicus' model, Kepler refined the model, keeping the relation between the nodes but simplifying the trajectories from complex epicycles to ellipses.

In such examples, the diagram works retrospectively to explore and solve existing problems (finding the simplest model explaining the observations of heavenly bodies). In other cases, diagram manipulation works prospectively facilitating new insights by redefining the terms of the problems to be solved. For instance, in Duncker's Radiation Problem (Duncker, 1945) participants face the task of eliminating a tumor with a radiation source without destroying the surrounding healthy tissue. The situation is presented in a diagram (see Figure 2). While most participants would often focus their attention on the tumor, a cue to the solution lies in attending to the spatially extended nature of the boundary: by hitting the tumor from many different angles with less intense radiation, the healthy tissue remains unaffected while radiation intensely cumulates on the tumor. This change in problem representation was found to be facilitated by an explicit diagrammatic manipulation: when the researchers visually reinforced the skin boundary (making it blink), the solution became more obvious and more participants reached the right solution (Grant \& Spivey, 2003). Both examples - the retrospective modeling of observations and the prospective solution to a problem - are illustrative of the gestalt notions of fixedness and insight (Köhler, 1925; Ohlsson, 1992). Fixedness 


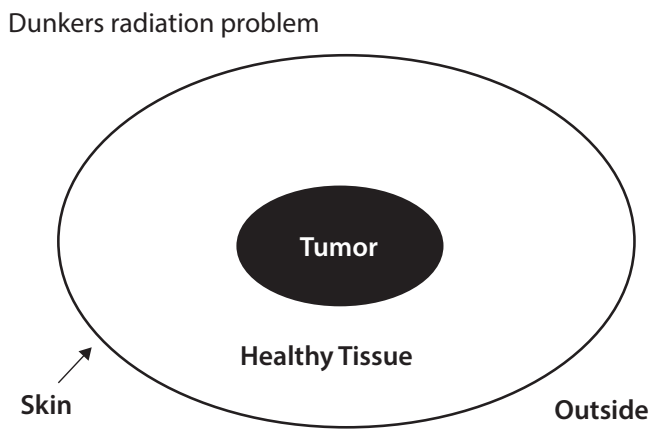

Figure 3. Reproduction of the diagram from Dunkers Radiation Problem. The diagram is accompanied by the following instruction: "Suppose you are a doctor faced with a patient who has an inoperable stomach tumor. You have at your disposal rays that can destroy human tissue when directed with sufficient intensity. How can you use these rays to destroy the tumor without destroying the surrounding healthy tissue?" (Duncker, 1945).

relates to the way that entrenched schematic representations obstruct the road to the right solution; for instance, the Greek "common knowledge" that all motion can be described by circular motions and the medieval "common knowledge" that the earth is the center of the universe, or, in Duncker's problem, the entrenched experience that radiation comes from one source. Insight, on the other hand, is when the entrenched knowledge is discarded for the right solution. Diagrams can afford insight because the solution is potentially present through a more or less simple manipulation. Exploratory diagram manipulations can help shifting attention to the right information and thus break loose of mental fixedness. Crucially, this manipulation can happen in a socially and even temporally distributed way: Copernicus relied on and simplified previous models, just like the participants in Grant \& Spivey's version of Duncker's problem could rely on the researchers' subtle diagrammatic manipulation.

As pointed out above, one of the central features of diagrams is their manipulability. While many types of symbolic representations (including e.g. traffic signs) represent meaning in fixed and static ways, diagrammatical representations are special in their affordances for dynamical interaction. Through their instantiations in manipulable media (e.g. drawings on a whiteboard, two or three dimensional models, scribbles or graphics) they invite for manual exploration: they can quite literally be poked, prodded, pulled apart and reassembled. By experimenting on the material aspects of the diagram, we can explore the consequences on the conceptual structures of the diagram-type with the potential of gaining new ideas, solutions and insights. In the following we consider different kinds of diagrammatical manipulations involved in problem solving. We can individuate two 
prototypical cases, one in which the diagram helps to generate information and one in which it is used to reduce and extract information.

\subsection{Manipulating diagrams to generate information}

Many simple problem-solving scenarios entail impoverished problem spaces in the sense that what is directly accessible is not enough to solve the problem. In order to find the optimal solution, the agent must generate or infer extra information that is not directly available. This can in principle be done by pure mental operations, however, creative thought that takes place entirely in the head is cognitive costly and heavily constrained by entrenched schemas, previous knowledge, shortterm memory, etc. (Cowley \& Vallée-Tourangeau, 2014). Often a more optimal strategy is to transform the search procedure into a manual activity of exploring the presentation-side of representational artifacts or diagrams. An abstract problem is thus turned into a very concrete activity of manual experimentation on the representational medium. In general, we suggest that a diagram is used to generate information in any case where there is a manipulation of the object of study and where this manipulation is necessary to access relevant (and new) information.

In classical examples of such diagram manipulation, auxiliary lines are added to form a geometrical proof (Peirce, 1976; Stjernfelt, 2007). For instance, proving that the sum of the angles in a triangle is 180 degrees cannot be achieved by just using the information we get from the figure of the triangle. The addition of particular extra lines is a way to generate new information that is critical for solving the case. A special issue here concerns how to chose the right auxiliaries among many possible (Shin, 2010). Closely related to these observations, Vallée-Tourangeau and colleagues demonstrated that the "degree of manipulability" of material mediation has a significant impact on performance in arithmetic and insight problem solving. In an arithmetic task, participants improved their efficiency in the task if the problem was presented in physical, manipulable tokens. The effect was most pronounced in tasks with highest complexity/most numbers (Vallée-Tourangeau, 2013). In a similar vein, performance in an insight problem solving task involving 'matchstick algebra problems' radically improved if participants were presented with actual matchsticks (rather than two-dimensional, figurative depictions) and were allowed to freely engage them (Knoblich, Ohlsson, Haider, \& Rhenius, 1999; Weller, Villejoubert, \& Vallée-Tourangeau, 2011). The introduction of more manipulable representations thus increases the likelihood of achieving insight.

But the principles of manipulating diagrams in order to generate additional information also apply to more mundane cases of problem solving. An illustrative example is provided in an experiment on the game of Tetris (Kirsh \& Maglio, 1994). In Tetris, players have to make quick decisions on how to rotate a falling tile 
in order to make it fit a socket. Here, the problem space is impoverished to the extent that the tile is initially only presented in one orientation and the task is to decide if to rotate it to another orientation before it falls into a socket. Interestingly, Kirsh and Maglio found that novice players generally relied on mental rotations to search for the optimal solution, while expert players performed more manual rotations. Manual rotation was shown to be a faster and more effective way to explore possibilities and inform decisions in regard to the final placement of the Tetris tiles. Along similar lines, in a Scrabble-like experiment, participants were instructed to form as many words as possible from a limited set of letter tiles. Again the finding was that participants increased their performance (produced more words) when allowed to manually manipulate letter tiles to assist their search (Maglio, Matlock, Raphaely, Chernicky, \& Kirsh, 1999).

What seems to be at stake here is that the manual manipulation of letter tiles introduces an element of unpredictability that potentially disrupts functional fixedness and other constraining biases. By the same logic we can speculate that this effect might be even enhanced in the case of the joint manipulation of public representations. When jointly engaging in epistemic explorations of a shared diagrammatic medium, individual participants are not only presented with the task of drawing the consequences of their own manipulations. They also witness manipulations that they might not have premeditated but from which they may be able to draw conclusions that the 'manipulator' had not envisioned. Examples of this phenomenon was observed by Bjørndahl and colleagues (2014) in groups jointly building LEGO models of their shared understanding of abstract concepts. Analyses of the group interactions motivated a taxonomy of different roles that the material representations would play in the joint epistemic processes. In some cases, LEGO blocks were used 'illustratively' to support the communication of already well-formed ideas. At other times, the concrete instantiation of an idea in LEGO blocks came to reveal unnoticed conceptual aspects and disagreements thus leading to collective examination, discussion, and elaborations. Lastly, it was observed how participants sometimes critically relied on the manual manipulation of representational materials to actually generate ideas. In these cases, participants experimented on and explored the LEGO blocks with the result of discovering new ideas and meaning potentials. In these cases the manual manipulation of the materials played an active role in shaping and catalyzing joint epistemic processes.

\subsection{Manipulating diagrams to extract information}

While in the preceding examples participants are confronted with impoverished problem spaces, other problem solving scenarios present us with the opposite challenge: there is, from the outset, too much information. These problems afford 
quite different types of diagrammatical reasoning: the solution lies rather in discarding unnecessary or irrelevant information in order to identify the core elements or relations. In other words diagrams work by abstraction.

For instance, when navigating a subway map the actual shape of trajectories of the metro train is not important. Only the station sequences and their possible connections matter. Consulting the first charts of the London metro, which depicted the full and detailed trajectories of the routes, indicates why this is not trivial insight. More contemporary metro charts (beginning with Harry Beck's

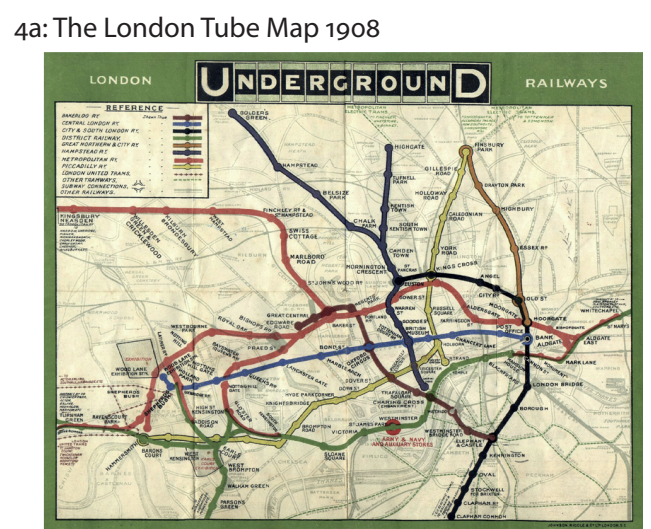

4b: The London Tube Map 1933

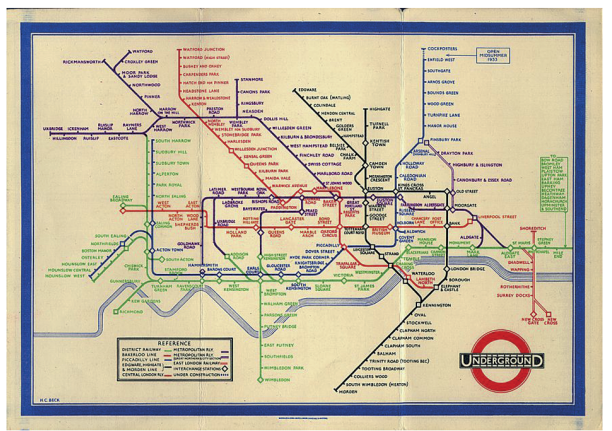

4c: The London Tube - Victoria Line

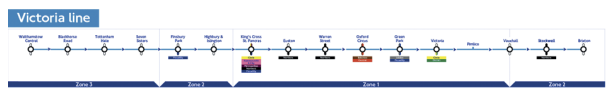

Figure 4. Three reproductions of the London Tube. 3a: an early version of the map (1908) that presents subway routes and stations retaining their relative topography. 3b: Harry Beck's diagrammatic reproduction of the tube map from 1933. This version of the map discards irrelevant details concerning route shapes and only presents a simplified topology. 3c: A diagram of the London Tube Victoria Line. Notice that this is even further abstracted away from the actual shape of the route by only representing the succession of stations equally distributed on a strait line. 
famous redesign of 1931) present the optimal representation of a fixed configuration abstracting away from irrelevant details concerning shapes of trajectories (see Figure 4, cf. also Atã, Bitarello, \& Queiroz, 2014).

Flow charts constitute another prototypical use of diagrams to extract information. Interestingly, they are indifferent to the underlying ontology of represented elements: they can represent an algorithm, the communication channels in an organization, the work process in a company, the energy flow in a heating system etc. By abstracting away specific embodied experiences and only representing the most relevant information in nodes and arrows, the flow chart affords easy manipulation and thus facilitates analysis, design or management of all kinds of processes.

The generality of this abstraction procedure lies in the reduction of sensory information in favor of a formal model. Following insights from cognitive semantics (Fauconnier \& Turner, 2002; Lakoff \& Johnson, 1999), this relies on the human capacity to selectively map structure from one domain to another. Andy Clark nicely illustrates this mapping of embodied experience into another representational format in a reinterpretation of Boysen and colleagues' work on Sheba and the treats (Boysen, Bernston, Hannan, \& Cacioppo, 1996; A. Clark, 2006b). In an experiment, the chimpanzee Sheba is presented with the choice of two plates of grapes. If she points to the plate with more grapes she gets the one with fewer and the other way around. Although trained on numerals, Sheba is caught in her immediate inclinations and just cannot seem to distract from the natural affordances of the grapes and keeps pointing to the larger plate. However, when symbolic labels are attached to the plates showing the number of grapes, suddenly Sheba solves the riddle and starts pointing to the smaller number to get the larger plate. Importantly, again the solution lies in the movement away from the concrete scenario by abstraction: The juicy and tasty appearance of the grapes is too strong an attraction, putting the property of quantity in the far background. By replacing them with a representation focused only on the quantity, Sheba is enabled to change problem representation and find the proper solution.

A similar case is found in a problem-solving task involving interconnected cogwheels (Alibali, Spencer, Knox, \& Kita, 2011; Dixon, Stephen, Boncoddo, \& Anastas, 2010; Schwartz, 1995). Here participants are presented with a series of interconnected gears and asked to infer the rotation direction of the last gear from the first. Again the task requires participants to abstract away from irrelevant information - the size, color, and arrangement of the cogwheels - and home in on a single critical parameter: the direction. While most participants confronted with the task initially conceive of the problem in mechanical, causal terms, the superior solution is simply to count the connected cogwheel to establish whether there is an equal or unequal number. This, again, is a movement towards higher abstraction: that is, mapping from the body domain to the domain of numbers. Accordingly, 
studies suggest that participants relying on more rich, manual gestural strategies are outperformed by participants resorting to more symbolic verbal strategies in the task (Alibali, et al., 2011).

\section{Distributed diagrammatic reasoning: The case of scientific diagrams}

By constructing and manipulating external representations, diagrammatic reasoning allows human cognitive processes to be distributed not only across multiple participants, but also across different time scales. A gestural diagram addressing how to find one's way in a cityscape may be functional over a time scale of minutes; the sketching of flow charts or similar diagrammatic representations on a whiteboard might assist joint problem-solving activities on a time scale of hours, while central scientific diagrams, on the other hand, may exist and evolve on a time scale of years. Finally, certain classical diagrams like the proofs of Pythagoras or Euclid or the diagram of planetary movements might count their existence in millennia. However, in all cases there is an interesting distinction between elementary rules that one has to comply with (the manipulations that are allowed and possible) and the different strategies used to solve a problem, which might vary considerably (Hintikka, 2007). While the first are more stable and generally belong to a larger time scale, independent of the particular experiments, solution strategies can be tried out and discovered by the participants on much smaller, online time scales. For instance, while the addition of auxiliary lines to form a proof in the geometry case above is an expression of an inventive local strategy, it still follows more general and stable rules for how to interact with and interpret lines and angles in geometry as a scientific discipline.

These points are particularly evident in scientific diagrammatic reasoning, as highlighted by the work of Bechtel and colleagues on the study of chronobiology, that is, of periodic phenomena in living organisms, such as circadian rhythms (Sheredos, Burnston, Abrahamsen, \& Bechtel, 2013). Burnston et al followed the use of diagrams as collected data turned into a submitted chronobiology paper (Burnston, Sheredos, Abrahamsen, \& Bechtel, 2015). Existing diagrams of the basic circadian mechanism in a cyanobacterium were employed to highlight poorly understood aspects of the mechanism and motivate new questions. This led to the creation of diagrams of the data exploring those questions. These graphs evolved radically during the months in which the data were analyzed and the paper written, with elements from different attempts being re-used and developed and irrelevant details omitted.

This process is by no means isolated to the individual lab and individual publication, but form part of a larger and distributed process of scientific discovery. 
Bechtel and Abrahamsen (2012) thus examined the representation of circadian rhythms across several decades of scientific publications, which preceded and inspired those investigated in the previous study. Different diagrammatic conventions are developed to answer different research directions: e.g. oscillations, actograms and phase response curves. Phase response curves, in particular, are shown to evolve over time between their introduction in 1958 and current use, as different researchers abstract from certain aspects, e.g. the specific time of day, to focus on more informative abstract relations, e.g. phase synchronization, thus developing new conventions and cognitive affordances. Thus, each individual investigation relies on the slowly evolving diagrammatic conventions, but also challenges and develops them. In this way, diagrams pervade and facilitate distributed forms of thinking and manipulating knowledge.

While traditional research in philosophy and history of science has privileged induction from experimental results and deduction from existing knowledge, diagrammatic reasoning seems to occupy a central position. Inspired by other domains, scientists attempt to organize their data and understanding of a topic in diagrammatic representations, which are manipulated to gain insight and lead to new experiments.

\section{Conclusion}

Taking as a starting point the foundational reflections of C.S. Peirce, we have outlined a taxonomy of different kinds of diagrammatical reasoning. Distinctions are substantiated by reference to empirical studies across history of ideas, cognitive science, experimental semiotics and sociology of science. Diagrammatic reasoning maps human experiences into a representational format, which allows for different kinds of operations and manipulations. Some of these serve the purpose of redescription: through diagrammatical visualizations, data can be explored and profiled in numerous ways, selectively highlighting different aspects of the referent. These operations are important for instance for communication purposes and seem involved in the evolution and refinement of conceptualization and communication systems on multiple time scales.

Other diagram manipulations rather serve different kinds of reasoning processes. These are cases where diagrams are manipulated and explored in order to gain new insights about the referent, for instance in the context of problem solving. We have suggested two main types of diagrammatical (theorematic) reasoning observed in response to different kinds of problems. Sometimes diagrams are manipulated in order to generate additional information critical for the solution to a problem, but not readily available at the outset. We add lines to a diagram, 
manually manipulate models and representations to escape cognitive fixedness and multiply strategies to exhaust a search space. In other cases diagrams provide a basic mechanism for abstraction: the diagram is thus a way of selectively extract and represent only the most relevant dimensions of an otherwise complex problem scenario. Rather than generate information, the diagram enables us to discard the complex sensory information that might prevent us from appreciating particularly important relations and mechanisms.

Diagrammatic reasoning is thus explorative, that is, a process of experiments and attempts, which enables us to profile information, escape fixedness and achieve new insights or creative solutions. Crucially, much of diagrammatic reasoning is active sketching and manipulation of external, public representational formats, which opens for collective diagrammatic reasoning. Its social nature is seen both on shorter time scales where diagrams make it possible for participants in real time to jointly construct, explore and reconfigure representational tokens until a satisfactory solution is obtained. But diagrams also work to distribute human reasoning on longer time scales: like any other object, a diagram developed in a particular field or community can be passed on and thus become subject of long term development and refinement as in the example with the planetary movements. This allows for the construction of cumulative and increasingly complex socio-cultural niches for human cognition (Tylén, et al., 2013).

In summary, we argue that diagrammatic reasoning plays a fundamental role in human thinking from everyday communications to the creation of mathematical theorems. In all cases the same basic cognitive dynamics seems at work: i) the fundamental ability to map human experiences onto a diagrammatic - often external - format; ii) which makes abstract relations perceivable; and iii) 'manipulable', thus enabling us to push, poke and mold our thoughts and meanings by working on their representational tokens; finally iv) their external/public nature permit collective thinking, communication and collaboration, sometimes over large timescales. Together these central aspects of diagrammatical reasoning make new and unprecedented modes of creative thinking and discovery possible.

\section{Acknowledgements}

This work is funded by the Danish Council for Independent Research | Humanities' project Joint Diagrammatical Reasoning in Language and The Interacting Minds Center, Aarhus University. 


\section{References}

Alibali, M. W., Spencer, R. C., Knox, L., \& Kita, S. (2011). Spontaneous gestures influence strategy choices in problem solving. Psychol Sci, 22(9), 1138-1144. DOI: 10.1177/0956797611417722

Atã, P., Bitarello, B., \& Queiroz, J. (2014). Iconic semiosis and representational efficiency in the London Underground Diagram. Cognitive Semiotics, 7(2), 177-190.

DOI: $10.1515 /$ cogsem-2014-0012

Bechtel, W., \& Abrahamsen, A. (2012). Diagramming phenomena for mechanistic explanation. Paper presented at the Proceedings of the 34th Annual Conference of the Cognitive Science Society.

Bjørndahl, J., Fusaroli, R., Østergaard, S., \& Tylén, K. (2014). Thinking together with material representations: Joint epistemic actions in creative problem solving. Cognitive Semiotics, 7(1), 103-123.

Bjørndahl, J., Fusaroli, R., Østergaard, S., \& Tylén, K. (2015). When agreeing is not enough. Interaction Studies, 16(3).

Bjørndahl, J. S., Fusaroli, R., Østergaard, S., \& Tylén, K. (2014). Thinking together with material representations: Joint epistemic actions in creative problem solving. Cognitive Semiotics, 7(1), 103-123.

Boysen, S. T., Bernston, G. G., Hannan, M. B., \& Cacioppo, J. T. (1996). Quantity-based interference and symbolic representations in chimpanzees (Pan troglodytes). Journal of experimental psychology. Animal behavior processes, 22(1), 76-76. DOI: 10.1037/0097-7403.22.1.76

Burnston, D., Sheredos, B., Abrahamsen, A., \& Bechtel, W. (2015). Scientists' Use of Diagrams in Developing Mechanistic Explanations, Pragmatics and Cognition. Pragmatics and Cognition., 22(2), 224-242

Cairo, A. (2012). The Functional Art: An introduction to information graphics and visualization. New Riders.

Clark, A. (2006a). Language, embodiment, and the cognitive niche. Trends Cogn Sci, 10(8), 370-374. DOI: 10.1016/j.tics.2006.06.012

Clark, A. (2006b). Material symbols. Philosophical psychology, 19(3), 291-307. DOI: $10.1080 / 09515080600689872$

Clark, A. (2008). Supersizing the mind: Embodiment, action, and cognitive extension. New York: Oxford University Press.

Clark, A., \& Chalmers, D. (1998). The extended mind. Analysis, 58(1), 7-19. DOI: 10.1093 /analys/58.1.7

Clark, H. H., \& Krych, M. A. (2004). Speaking while monitoring addressees for understanding. $62-81$

Cowley, S. J., \& Vallée-Tourangeau, F. d. r. (Eds.). (2014). Cognition Beyond the Brain: Computation, Interactivity and Human Artifice (Vol. 17). Springer.

Dale, R., Fusaroli, R., Duran, N., \& Richardson, D. C. (2013). The self-organization of human interaction. Psychology of Learning and Motivation, 59, 43-95.

DOI: 10.1016/B978-0-12-407187-2.00002-2

Dixon, J. A., Stephen, D. G., Boncoddo, R., \& Anastas, J. (2010). The self-organization of cognitive structure. Psychology of Learning and Motivation, 52, 343-384.

DOI: 10.1016/S0079-7421(10)52009-7

Duncker, K. (1945). The Structure and Dynamics of Problem-Solving Processes. Psychological Monographs, 58(5), 1-112. DOI: 10.1037/h0093599 
Fauconnier, G., \& Turner, M. (2002). The way we think: Conceptual blending and the mind's hidden complexities. New York: Basic Books.

Fay, N., Garrod, S., Roberts, L., \& Swoboda, N. (2010). The interactive evolution of human communication systems. Cogn Sci, 34(3), 351-386. DOI: 10.1111/j.1551-6709.2009.01090.x

Fusaroli, R., Bahrami, B., Olsen, K., Rees, G., Frith, C. D., Roepstorff, A., \& Tylén, K. (2012). Coming to terms: an experimental quantification of the coordinative benefits of linguistic interaction. Psychological Science, 23, 931-939. DOI: 10.1177/0956797612436816

Fusaroli, R., Gangopadhyay, N., \& Tylén, K. (2014). The dialogically extended mind: Making a case for language as skilful intersubjective engagement. Cognitive Systems Research, 29-30, 31-39. DOI: 10.1016/j.cogsys.2013.06.002

Fusaroli, R., Raczaszek-Leonardi, J., \& Tylén, K. (2014). Dialog as interpersonal synergy. New Ideas in Psychology, 32, 147-157. DOI: 10.1016/j.newideapsych.2013.03.005

Fusaroli, R., \& Tylén, K. (2012). Carving Language for Social Coordination: a dynamic approach. Interaction Studies, 13, 103-123. DOI: 10.1075/is.13.1.07fus

Fusaroli, R., \& Tylén, K. (in press). Investigating conversational dynamics: Interactive alignment, Interpersonal synergy, and collective task performance. Cognitive Science.

Galantucci, B., \& Garrod, S. (2010). A new approach for studying the emergence and the evolution of human communication. Interaction studies, 11(1), 1-13. DOI: 10.1075/is.11.1.01gal

Garrod, S., \& Doherty, G. (1994). Conversation, co-ordination and convention: An empirical investigation of how groups establish linguistic conventions. Cognition, 53(3), 181-215. DOI: $10.1016 / 0010-0277(94) 90048-5$

Garrod, S., Fay, N., Lee, J., Oberlander, J., \& MacLeod, T. (2007). Foundations of Representation: Where Might Graphical Symbol Systems Come From? Cognitive Science, 31(6), 961-987. DOI: $10.1080 / 03640210701703659$

Grant, E. R., \& Spivey, M. J. (2003). Eye movements and problem solving guiding attention guides thought. Psychological Science, 14(5), 462-466. DOI: 10.1111/1467-9280.02454

Healey, P. G., Swoboda, N., Umata, I., \& King, J. (2007). Graphical language games: Interactional constraints on representational form. Cognitive Science, 31(2), 285-309.

Hintikka, J. (1983). The game of language: Studies in game-theoretical semantics and its applications. Springer.

Hintikka, J. (2007). Socratic Epistemology: Explorations of Knowledge-Seeking by Questioning. Cambridge: Cambridge University Press. DOI: 10.1017/CBO9780511619298

Hutchins, E. (1995). Cognition in the Wild. Cambridge, Mass.: MIT Press.

Hutchins, E. (2005). Material anchors for conceptual blends. Journal of Pragmatics, 37(10), 1555-1577. DOI: 10.1016/j.pragma.2004.06.008

Hutchins, E. (2010). Cognitive Ecology. Topics in Cognitive Science, 2(4), 705-715. DOI: $10.1111 /$ j.1756-8765.2010.01089.x

Karmiloff-Smith, A. (1995). Beyond modularity: A developmental perspective on cognitive science. MIT press.

Kirsh, D., \& Maglio, P. (1994). On distinguishing epistemic from pragmatic action. Cognitive Science, 18(4), 513-549. DOI: 10.1207/s15516709cog1804_1

Knoblich, G., Ohlsson, S., Haider, H., \& Rhenius, D. (1999). Constraint relaxation and chunk decomposition in insight problem solving. Journal of Experimental Psychology: Learning, Memory, and Cognition, 25(6), 1534. DOI: 10.1037/0278-7393.25.6.1534

Köhler, W. (1925). Komplextheorie und Gestalttheorie. Psychological Research, 6(1), 358-416. DOI: $10.1007 / \mathrm{BF} 00444185$ 
Lakoff, G., \& Johnson, M. (1999). Philosophy in the flesh: The embodied mind and its challenge to western thought. New York: Basic Books.

Maglio, P. P., Matlock, T., Raphaely, D., Chernicky, B., \& Kirsh, D. (1999). Interactive skill in scrabble. Paper presented at the Proceedings of the Twenty First Annual Conference of the Cognitive Science Society.

Ohlsson, S. (1992). Information-processing explanations of insight and related phenomena. Advances in the psychology of thinking, chap. 1, 1-44.

Peirce, C. S. (1931). Collected Papers of Charles Sanders Peirce: Vols. 1-6 (C. Hartshorne \& P. Weiss, eds.). Cambridge, Mass.: Harvard University Press.

Peirce, C. S. (1976). The New Elements of Mathematics by C.S. Peirce, edited by Carolyn Eisele. Walter De Gruyter.

Peirce, C. S. (1981). Writings of Charles S. Peirce : a chronological edition. Bloomington: Indiana University Press.

Peirce, C. S. (1998). Charles S. Peirce : the essential writings. Amherst, N.Y.: Prometheus Books.

Schwartz, D. L. (1995). The emergence of abstract representations in dyad problem solving. Journal of the Learning Sciences, 4(3), 321-354. DOI: 10.1207/s15327809jls0403_3

Sheredos, B., Burnston, D., Abrahamsen, A., \& Bechtel, W. (2013). Why do biologists use so many diagrams? Philosophy of Science, 80(5), 931-944. DOI: 10.1086/674047

Shin, S.-J. (2010). Peirce's two ways of abstraction. In M. Moore (Ed.), New Essays on Peirce's Mathematical Philosophy. Chicago and La Salle: Open Court.

Stjernfelt, F. (2000). Diagrams as centerpiece of a Peircean epistemology. Transactions of the Charles S. Peirce Society, XXXVI(3), 357-392.

Stjernfelt, F. (2007). Diagrammatology: An investigation on the borderlines of phenomenology, ontology, and semiotics. New York: Springer.

Stjernfelt, F. (2014). Natural Propositions: The Actuality of Peirce's Doctrine of Dicisigns. Docent Press.

Tek, S., Mesite, L., Fein, D., \& Naigles, L. (2014). Longitudinal analyses of expressive language development reveal two distinct language profiles among young children with autism spectrum disorders. Journal of autism and developmental disorders, 44(1), 75-89. DOI: $10.1007 / \mathrm{s} 10803-013-1853-4$

Tylén, K., Fusaroli, R., Bundgaard, P., \& Østergaard, S. (2013). Making sense together: A dynamical account of linguistic meaning making. Semiotica, 194, 39-62.

Vallée-Tourangeau, F. (2013). Interactivity, efficiency, and individual differences in mental arithmetic. Experimental psychology, 60(4), 302. DOI: 10.1027/1618-3169/a000200

Weller, A., Villejoubert, G., \& Vallée-Tourangeau, F. (2011). Interactive insight problem solving. Thinking \& Reasoning, 17(4), 424-439. DOI: 10.1080/13546783.2011.629081

Yau, N. (2011). Visualize this: the FlowingData guide to design, visualization, and statistics. John Wiley \& Sons. 


\section{Authors' addresses}

Riccardo Fusaroli, Kristian Tylén, Johanne Bjørndahl, Svend Østergaard Center for Semiotics

Jens Chr Skous Vej 2

8000 Aarhus

Denmark

fusaroli@dac.au.dk; kristian@dac.au.dk; semjsb@dac.au.dk; semsvend@dac.au.dk
Frederik Stjernfelt

Institut for Kunst og Kulturvidenskab Karen Blixensvej 1

2300 København S

Denmark

stjern@hum.ku.dk 